\title{
Insertion Loss Function Synthesis of Maximally Flat Parallel-Coupled Line Bandpass Filters
}

\author{
Kuo-Sheng Chin and Jen-Tsai Kuo, Senior Member, IEEE
}

\begin{abstract}
Insertion loss (IL) functions are derived for synthesis of microstrip parallel-coupled line bandpass filters with maximally flat responses. The derivation is performed by successively multiplying the $A B C D$ matrices of all coupled stages. Simultaneous equations for determining linewidth and line spacing of the coupled stages are established by total $Q\left(Q_{T}\right)$ of the filter specification and comparing the IL function with the canonical form. The results are provided for filters of order $N \leq 6$. Two filters with fractional bandwidths $\Delta=30 \%$ are synthesized and demonstrated by simulation using a full-wave software package. In addition, two filters with $\Delta=40 \%$ and $50 \%$ are fabricated. Tapped line inputs are employed to these two circuits since some linewidths or gaps are beyond the fabrication resolution. The measured results show very good agreement with the theoretical responses.
\end{abstract}

Index Terms-Insertion loss (IL) function, maximally flat response, parallel-coupled line filter, $Q$-distribution method, tapped line.

\section{INTRODUCTION}

$\mathbf{T}$ HE parallel-coupled line filter has been widely used in microwave communication systems for at least three decades [1]-[5]. It is popular since it has an easy synthesis procedure and a wide range of realizable bandwidths (BWs). In a conventional design, approximate synthesis formulas have been well documented for determining dimensions of each coupled stage [4], [5]. One of the key steps is to establish an equivalence of a coupled stage at design frequency $\left(f_{o}\right)$ to a two-port network consisting of two quarter-wave transmission line sections with an admittance inverter in between [5]. Since the admittance inverter is assumed independent of frequency, the exact equivalence is valid only at $f_{o}$. Thus, the formulas are accurate only for bandpass filters (BPFs) with a relative small BW.

It is because the coupling coefficient of each stage decreases as frequency moves away from $f_{o}$, BPFs synthesized based on the conventional method will have a fractional BW $\Delta$ less than specification. The BW decrement deteriorates as filter order or designed BW is increased. As reported in [6], when filter order $N=3$ and $\Delta=35 \%$, the synthesized circuit has only $\Delta=$ $30 \%$. When $\Delta=50 \%$, the realized BWs are only $41 \%$ and $38 \%$ for $N=3$ and $N=5$, respectively. For recovering the BW decrement, new formulas for determining $Z_{o e}$ and $Z_{o o}$ of each coupled stage have been derived in [6] for synthesizing relatively wide-band filters. In this way, the realized BWs can be

Manuscript received January 26, 2005, revised April 4, 2005. This work was supported in part by the National Science Council, Taiwan, R.O.C., under Grant NSC 93-2213-E-009-095 and Grant NSC 93-2752-E-009-002-PAE.

The authors are with the Department of Communication Engineering, National Chiao Tung University, Hsinchu 300, Taiwan, R.O.C. (e-mail: jtkuo@cc.nctu.edu.tw).

Digital Object Identifier 10.1109/TMTT.2005.855355

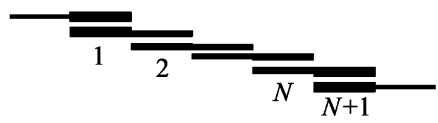

Fig. 1. Layout of an $N$ th-order parallel-coupled line filter.

greatly improved, but the BW decrement is still not completely resolved. For example, when $\Delta=50 \%$ is given, the new designs still have only $48.2 \%$ and $44 \%$ for $N=3$ and $N=5$, respectively.

Some methods have been proposed to design filters with accurate passband responses. In [7], insertion loss (IL) functions are derived for maximally flat filters with short-circuited quarterwave stubs. The $Q$ distribution method in [8] can provide accurate solutions to filters with narrow and wide BWs. Entire procedure for finding the $Q$ distribution includes choosing the number of sections, creating a composite $A B C D$ matrix, and solving individual admittance values of the resonators. For direct-coupled microwave filters of 2-12 resonant elements having $\Delta=$ $10 \% \sim 43 \%$, the theoretical results in [9] have good agreement with computed responses.

In this paper, the IL function of a parallel-coupled BPF is derived for synthesizing maximally flat responses. Based on the derived function, simultaneous conditions for determining dimensions of all coupled stages are provided. In the following, Section II shows the derivation for filters of order $N \leq 6$. Section III presents results of two filters to demonstrate the formulation and synthesis. In realizing two additional relatively wide-band filters, pattern resolution of certain stages exceeds our fabrication limits. Thus, in Section IV, tapped lines are designed to resolve this problem. Measured responses are compared with EM simulation and theoretical predictions. Finally, Section V draws the conclusion.

\section{IL FUNCTION}

For the $N$ th-order parallel-coupled microstrip filter in Fig. 1, let the generator and load impedances be identical and normalized to unity. Since a maximally flat response is assumed, the circuit layout is symmetric about its center and, when it is characterized by a composite $A B C D$ matrix, $A=D$ holds. It can be shown that the IL function can be written as [4]

$$
\frac{P_{o}}{P_{L}}=1+\left[\frac{j(B-C)}{2}\right]^{2}=1+\left(\Omega^{N}\right)^{2}
$$

where $j=\sqrt{-1}, N$ is order, $P_{o}$ is power available from source, and $P_{L}$ is power delivered to load. 


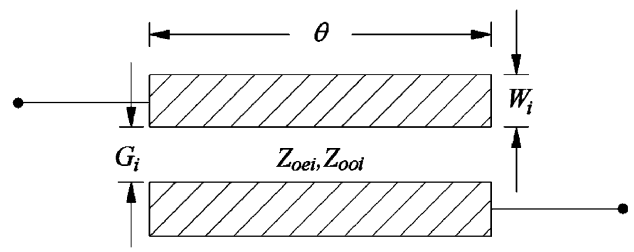

Fig. 2. $i$ th coupled stage with linewidth $W_{i}$ and gap $G_{i} . Z_{\text {oei }}$ and $Z_{\text {ooi }}$ are its even- and odd-mode characteristic impedances, respectively.

Of an $N$ th-order filter, the impedance matrix elements of the $i$ th coupled stage in Fig. 2 can be derived [5] as

$$
\begin{aligned}
& Z_{11 i}=Z_{22 i}=-\frac{j}{2}\left(Z_{o e i}+Z_{o o i}\right) \cot \theta \\
& Z_{12 i}=Z_{21 i}=-\frac{j}{2}\left(Z_{\text {oei }}-Z_{\text {ooi }}\right) \csc \theta
\end{aligned}
$$

where $\theta$ is its electrical length and $Z_{o e i}$ and $Z_{o o i}$ are the characteristic impedances of the even and odd modes, respectively. Here, the even- and odd-mode phase velocities for all coupled stages are assumed identical. From (2), the $A B C D$ matrix can be written as

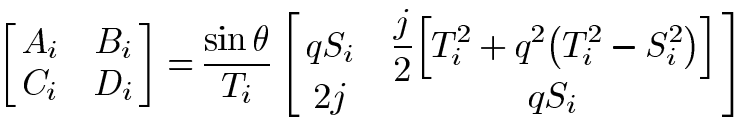

$$
\begin{aligned}
& q=\cot \theta \\
& S_{i}=\frac{Z_{o e i}+Z_{o o i}}{Z_{o}} \\
& T_{i}=\frac{Z_{o e i}-Z_{o o i}}{Z_{o}} .
\end{aligned}
$$

The composite $A B C D$ matrix of an $N$ th-order filter can be obtained by successively multiplying the $N+1 A B C D$ matrices as follows:

$$
\begin{aligned}
{\left[\begin{array}{ll}
A & B \\
C & D
\end{array}\right]_{N}=\left[\begin{array}{ll}
A_{1} & B_{1} \\
C_{1} & D_{1}
\end{array}\right]\left[\begin{array}{ll}
A_{2} & B_{2} \\
C_{2} & D_{2}
\end{array}\right] } \\
\\
\ldots\left[\begin{array}{ll}
A_{N+1} & B_{N+1} \\
C_{N+1} & D_{N+1}
\end{array}\right] .
\end{aligned}
$$

As $N$ becomes large, the result of (4) can be very tedious and complicated. If the matrix entries are expressed in terms of $q$, however, the results become much simpler. Substituting (3a) for all stages into (4) yields

$$
\left[\begin{array}{ll}
A & B \\
C & D
\end{array}\right]_{N}=\frac{\sin ^{N+1} \theta}{T_{1} T_{2} \cdots T_{N+1}} \times\left[\begin{array}{ll}
f_{a}(q) & f_{b}(q) \\
f_{c}(q) & f_{d}(q)
\end{array}\right]
$$

where $f_{a}(q), f_{b}(q), f_{c}(q)$, and $f_{d}(q)$ are in $(5 \mathrm{~b})$ and $(5 \mathrm{c})$, shown at the bottom of this page. The coefficients of each polynomial are functions of $S_{i}$ and $T_{i}$. The object of (5) is to find conditions for determining $Z_{o e i}$ and $Z_{o o i}$ and, hence, geometries of the coupled stages.

\section{A. First-Order Filters}

When $N=1$, the composite $A B C D$ matrix is a product of two identical $A B C D$ matrices. It can be derived that

$$
\begin{aligned}
\frac{j(B-C)}{2}=\frac{1}{T_{1}^{2}}[\sin \theta \cos \theta( & \left.2 S_{1}-\frac{1}{2} S_{1}^{3}\right) \\
& \left.+\frac{\cos \theta}{\sin \theta} \frac{S_{1}}{2}\left(S_{1}^{2}-T_{1}^{2}\right)\right] .
\end{aligned}
$$

Comparing (6) with the canonical form (1), we have

$$
2 S_{1}-\frac{1}{2} S_{1}^{3}=0 \text { or } S_{1}=2 .
$$

Substituting (7) into (6) yields the IL function

$$
\frac{P_{o}}{P_{L}}=1+\left[\frac{\cos \theta}{\sin \theta}\right]^{2}\left[\frac{S_{1}\left(S_{1}^{2}-T_{1}^{2}\right)}{2 T_{1}^{2}}\right]^{2} .
$$

The condition for solving $T_{1}$ can be obtained by imposing the given 3-dB BW to (8). This will be addressed later.

\section{B. Third-Order Filters}

For a third-order filter, $S_{1}=S_{4}, S_{2}=S_{3}, T_{1}=T_{4}$, and $T_{2}=T_{3}$. The composite $A B C D$ matrix can be obtained by multiplying the $A B C D$ matrices of the leading two stages, and post-multiplying the resulted matrix by itself with indices 1 and 2 being interchanged. The result can be written as

$$
\begin{aligned}
& \frac{j(B-C)}{2} \\
& =\frac{-1}{2 T_{1}^{2} T_{2}^{2}}\left[\begin{array}{l}
\sin \theta \cos \theta\left(h_{1}\right)+\sin \theta \cos ^{3} \theta\left(h_{2}-h_{1}-h_{3}\right) \\
\left.\quad+\frac{\cos ^{3} \theta}{\sin \theta}\left(h_{3}\right)\right]
\end{array}\right.
\end{aligned}
$$

where

$$
\begin{aligned}
h_{1}= & 4 T_{2}^{2}\left(S_{1}+S_{2}\right)-T_{1}^{2}\left(S_{1} T_{2}^{2}+S_{2} T_{1}^{2}\right) \\
h_{2}= & -2 T_{1}^{2}\left(S_{1} T_{2}^{2}+S_{2} T_{1}^{2}\right)+\left(S_{1}+S_{2}\right) \\
& \times\left(T_{2}^{2} S_{1}^{2}+2 T_{1}^{2} S_{1} S_{2}+4 T_{2}^{2}-4 S_{2}^{2}-4 S_{1} S_{2}\right) \\
h_{3}= & \left(T_{1}^{2}-S_{1}^{2}\right) S_{1} S_{2}^{2}-S_{2}\left(T_{1}^{2}-S_{1}^{2}\right)^{2}+\left(T_{2}^{2}-S_{2}^{2}\right) \\
& \times\left[S_{1}^{2} S_{2}-S_{1} T_{1}^{2}+S_{1}^{3}\right] .
\end{aligned}
$$

Matching (9a) with the canonical form (1), we reserve only the $\cos ^{3} \theta / \sin \theta$ term, i.e., enforce $h_{1}=0$ and $h_{2}=h_{3}$, to

$$
\begin{aligned}
& {\left[\begin{array}{ll}
f_{a}(q) & f_{b}(q) \\
f_{c}(q) & f_{d}(q)
\end{array}\right]=\left[\begin{array}{cc}
a_{0}+q^{2} a_{2}+\cdots+q^{N+1} a_{N+1} & \frac{j}{2}\left[q b_{1}+q^{3} b_{3}+\cdots+q^{N+2} b_{N+2}\right] \\
2 j\left[q c_{1}+q^{3} c_{3}+\cdots+q^{N} c_{N}\right] & d_{0}+q^{2} d_{2}+\cdots+q^{N+1} d_{N+1}
\end{array}\right] \quad N=\text { odd }} \\
& {\left[\begin{array}{ll}
f_{a}(q) & f_{b}(q) \\
f_{c}(q) & f_{d}(q)
\end{array}\right]=\left[\begin{array}{cc}
q a_{1}+q^{3} a_{3}+\cdots+q^{N+1} a_{N+1} & \frac{j}{2}\left[b_{0}+q^{2} b_{2}+\cdots+q^{N+2} b_{N+2}\right] \\
2 j\left[c_{0}+q^{2} c_{2}+\cdots+q^{N} c_{N}\right] & q d_{1}+q^{3} d_{3}+\cdots+q^{N+1} d_{N+1}
\end{array}\right] \quad N=\text { even }}
\end{aligned}
$$


TABLE I

MaXimally Flat CONDITIONS FOR $N=1 \sim 6$

\begin{tabular}{|c|c|c|}
\hline $\boldsymbol{N}$ & Maximally Flat Conditions & $\begin{array}{l}\text { Degree of } \\
\text { Freedom }\end{array}$ \\
\hline $\mathbf{1}$ & $S_{1}=2$ & 0 \\
\hline 2 & $\begin{array}{l}S_{1}=2 \\
2 T_{2}=T_{1}^{2}\end{array}$ & 1 \\
\hline 3 & $\begin{array}{l}S_{1}=2 \\
4 T_{2}^{2}\left(S_{1}+S_{2}\right)=T_{1}^{2}\left(S_{1} T_{2}^{2}+S_{2} T_{1}^{2}\right)\end{array}$ & 1 \\
\hline 4 & $\begin{array}{l}S_{1}=2 \\
2 T_{2}^{2}=T_{1}^{2} T_{3} \\
{\left[2 T_{3}\left(S_{1}+S_{2}\right)+T_{1}^{2}\left(S_{2}+S_{3}\right)\right]^{2}=2 T_{1}^{2}\left[T_{1}^{2}\left(S_{2}+S_{3}\right)^{2}+T_{2}^{2} S_{1}\left(S_{2}+S_{3}\right)+T_{3}^{2} S_{1}\left(S_{1}+S_{2}\right)\right]}\end{array}$ & 2 \\
\hline 5 & $\begin{array}{l}S_{1}=2 \\
4 T_{2}^{2}\left[T_{3}^{2}\left(S_{1}+S_{2}\right)+S_{3} T_{2}^{2}\right]=T_{1}^{2} T_{3}^{2}\left[S_{1} T_{2}^{2}+T_{1}^{2}\left(S_{2}+S_{3}\right)\right] \\
8 T_{2}^{2} S_{3}\left(S_{1}+S_{2}\right)+4 T_{3}^{2}\left(S_{1}+S_{2}\right)^{2}=T_{1}^{2}\left[T_{1}^{2} S_{3}\left(S_{2}+S_{3}\right)+2 T_{2}^{2} S_{1} S_{3}+2 T_{3}^{2} S_{1}\left(S_{1}+S_{2}\right)\right]\end{array}$ & 2 \\
\hline 6 & 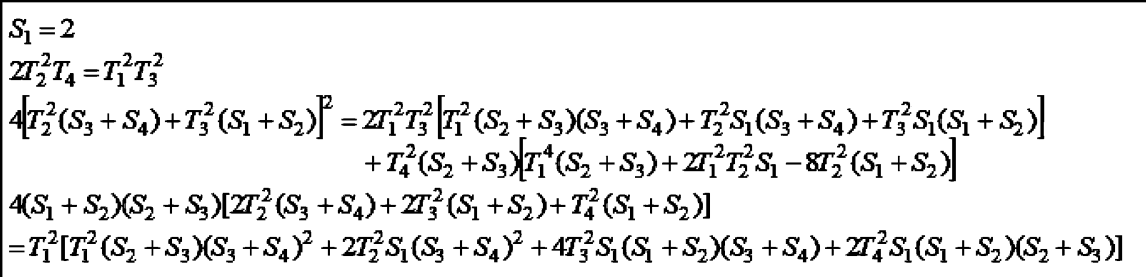 & 3 \\
\hline
\end{tabular}

eliminate the dependence of the IL function on $\sin \theta \cos \theta$ and $\sin \theta \cos ^{3} \theta$. It leads to the following two conditions:

$$
\begin{aligned}
S_{1} & =2 \\
4 T_{2}^{2}\left(S_{1}+S_{2}\right) & =T_{1}^{2}\left(S_{1} T_{2}^{2}+S_{2} T_{1}^{2}\right) .
\end{aligned}
$$

Inserting (10b) and (9d) into (9a) yields

$$
\frac{P_{o}}{P_{L}}=1+\left[\frac{\cos ^{3} \theta}{\sin \theta}\right]^{2}\left[\frac{2 S_{2}\left(S_{1}+S_{2}\right)\left[\left(S_{1}+S_{2}\right)-T_{1}^{2}\right]}{T_{1}^{2} T_{2}^{2}}\right]^{2} .
$$

The variable $S_{1}$ is purposely kept in (11) since it is useful in expressing the IL function in a general form. Note that there are four unknowns to be determined by only three equations, i.e., (10a), (10b), and (11) from the given BW. Thus, we have one degree of freedom in choosing the circuit dimensions.

\section{IL Function of a Filter of Order $N \leq 6$}

For an $N$ th-order filter, the IL function can be derived in a similar fashion. The simultaneous equations for solving $S_{i}$ and $T_{i}$ are obtained by saving the $\cos ^{N} \theta / \sin \theta$ term and enforcing coefficients for all other terms to zero. It is found that a general expression exists for the IL functions of order $N \leq 6$

$$
\begin{aligned}
\frac{P_{o}}{P_{L}} & =1+\left[\frac{\cos ^{N} \theta}{\sin \theta}\right]^{2}\left[K_{N}\right]^{2} \\
K_{N} & =\left|\frac{\prod_{i=1}^{N}\left(S_{i}+S_{i+1}\right)-\prod_{i=1}^{N-1}\left(S_{i}+S_{i+1}\right) T_{1}^{2}}{T_{1} T_{2} \cdots T_{N+1}}\right| .
\end{aligned}
$$

At the same time, the simultaneous conditions for determining $S_{i}$ and $T_{i}$ are listed in Table I. It is found that $S_{1}=2$ for each $N$. Note that total number of unknowns for an $N$ th-order filter is $N+1$ for odd $N$ and $N+2$ for even $N$. As shown in Table I, only $[N / 2]+2$ conditions are obtained, including the condition specified by the BW. Here, $[N / 2]$ is an integer by truncating $N / 2$. It can be seen that number of equations is less than that of unknowns when $N \geq 2$. For example, when $N=6$, eight variables have to be found for four of seven coupled stages. Three free dimensions exist since these variables are specified by only five equations. This under-determined feature is very helpful for circuit realization since both linewidth and gap size of coupled microstrips have resolution limits in fabrication. This will be discussed in Section IV.

\section{D. $Q_{T}$ Condition and the 3- $d B B W$}

For a maximally flat filter, the total $Q\left(Q_{T}\right)$ and $3-\mathrm{dB} \mathrm{BW}$ is related by

$$
Q_{T}=\frac{\omega_{0}}{\omega_{2}-\omega_{1}}
$$

where $\omega_{0}$ is the design frequency, and $\omega_{1}$ and $\omega_{2}$ are the $3-\mathrm{dB}$ cutoff frequencies specified by

$$
\left.\frac{P_{L}}{P_{o}}\right|_{\omega_{1}, \omega_{2}}=\frac{1}{2} .
$$

Thus, the electrical length $\theta$ can be written in terms of $Q_{T}$ as

$$
\left.\theta\right|_{\omega_{1}, \omega_{2}}=\frac{\pi}{2}\left(1 \pm \frac{1}{2 Q_{T}}\right)
$$




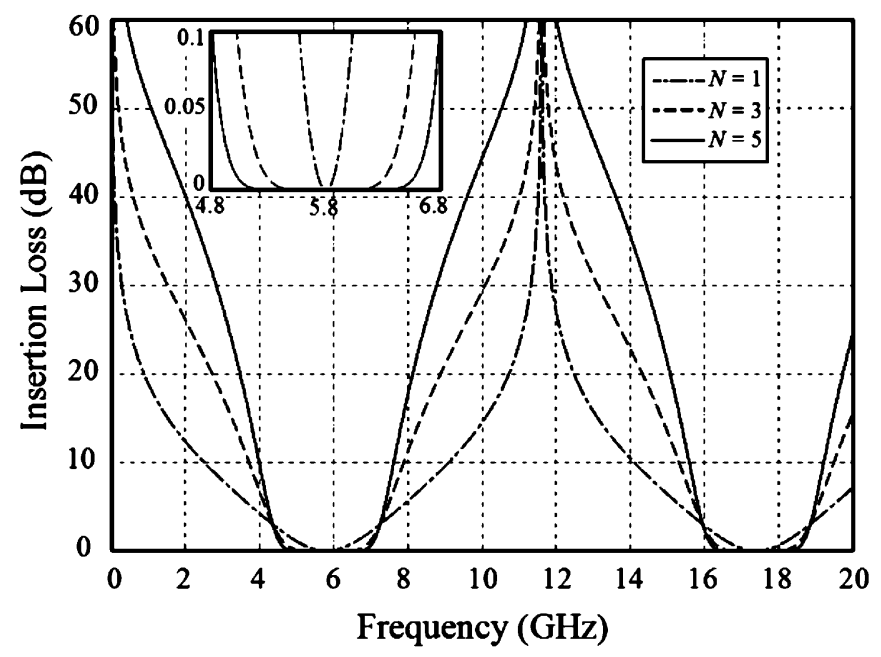

Fig. 3. Calculated maximally flat responses for $N=1,3$, and $5 . \Delta=50 \%$, $f_{o}=5.8 \mathrm{GHz}$.

and $K_{N}$ in (12) can be derived as

$$
\left.K_{N}\right|_{\omega_{1}, \omega_{2}}=\left|\frac{\sin \theta}{\cos ^{N} \theta}\right|_{\omega_{1}, \omega_{2}} .
$$

This is called the $Q_{T}$ condition herein. For demonstration, based on (12) and (16), Fig. 3 plots the calculated maximally flat responses for $N=1,3$, and 5 with $\Delta=50 \%$ and $f_{o}=5.8 \mathrm{GHz}$. The inserted frame shows the detailed passband performance. The synthesis method can be applied to Chebyshev filters as well. When $N=3$, the expressions of $h_{1}, h_{2}$, and $h_{3}$ in (9) are then specified by constants associated with the ripple level. Detailed results will be reported later in another form.

\section{Two EXAMPLES}

A third-order filter $\alpha$ and a sixth-order filter $\beta$ are synthesized at $f_{o}=5.8 \mathrm{GHz}$ with $\Delta=30 \%$ for validating the formulation. Simulated results via IE3D ${ }^{1}$ are presented for both circuits, while measurements are further performed for filter $\alpha$.

1) Filter $\alpha$ : When $N=3$, the $Q_{T}$ condition (16) gives

$$
2 S_{2}\left(2+S_{2}\right)\left[\left(2+S_{2}\right)-T_{1}^{2}\right]=\left|\frac{\sin \theta}{\cos ^{3} \theta}\right| T_{1}^{2} T_{2}^{2}
$$

where (10a) is used and, from (15), $\theta=1.3352 \mathrm{rad}$. Inserting (10b) into (17) yields

$$
\begin{aligned}
8\left(2+S_{2}\right)^{3}-12\left(2+S_{2}\right)^{2} T_{1}^{2}+4 & \left(2+S_{2}\right) T_{1}^{4} \\
& -\left|\frac{\sin \theta}{\cos ^{3} \theta}\right| T_{1}^{6}=0 .
\end{aligned}
$$

There is one degree of freedom in finding the solution. Fig. 4 plots the solutions of $S_{2}$ and $T_{2}$ for $T_{1}$ ranging from 0.9 to 1.3 . Referring to (3c) and (3d), we have $Z_{o e i}=\left(S_{i}+T_{i}\right) \times Z_{o} / 2$ and $Z_{\text {ooi }}=\left(S_{i}-T_{i}\right) \times Z_{o} / 2$. Obviously, not all roots shown in Fig. 4 are realizable using the standard microstrip technology. Realizable $Z_{o e i}$ and $Z_{o o i}$ depend on structural parameters, and obviously $Z_{o}$ is the dominant factor. Suppose that the filters are designed on a substrate with $\varepsilon_{r}=10.2$ and thickness $d=$

${ }^{1}$ IE3D Simulator, Zeland Software Inc., Freemont, CA, Jan. 1997.

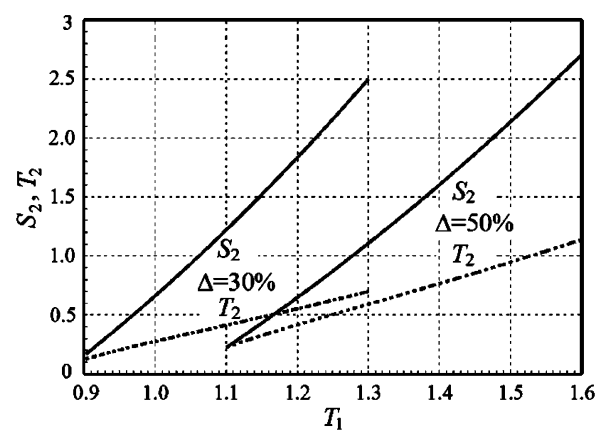

Fig. 4. Possible roots for $S_{2}$ and $T_{2}$ with respect to $T_{1}$ for a third-order filter with $\Delta=30 \%$ and $\Delta=50 \%$.

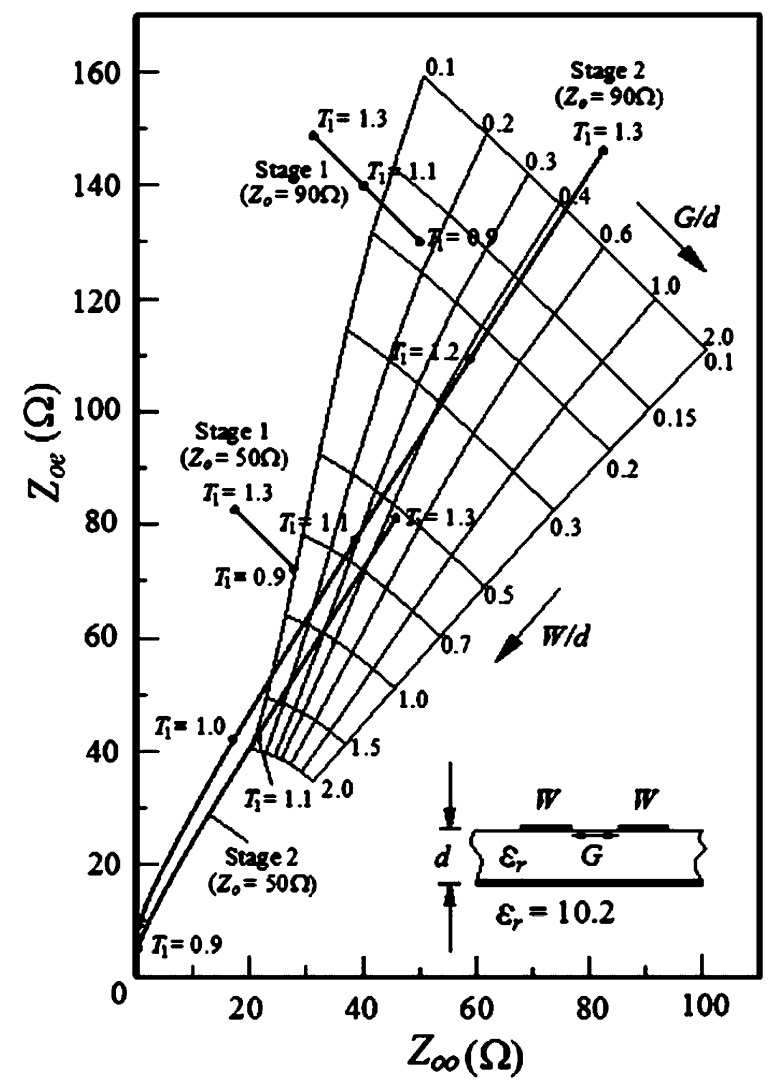

Fig. 5. Root loci for $Z_{o e}$ and $Z_{o o}$ of the first and second stages of filter $\alpha$ when $Z_{o}=50 \Omega$ and $90 \Omega$.

$1.27 \mathrm{~mm}$. According to resolution of our fabrication facilities, $G / d$ and $W / d$ must be no less than 0.1. When $Z_{o}=50 \Omega$ and $90 \Omega, Z_{o e}$ and $Z_{o o}$ for the first and second stages are plotted together with the design graph in Fig. 5. As $T_{1}$ is increased, values of $Z_{o e 1}, Z_{o e 2}$, and $Z_{o o 2}$ increase, while that of $Z_{o o 1}$ decreases. If $Z_{o}=50 \Omega$ is used, the gap size $G / d$ for stage 1 will be no larger than 0.1 . If both stages are required to have $G / d \geq 0.1$, for $Z_{o}=90 \Omega$, the value of $T_{1}$ must be between 1.03 and 1.06. Therefore, the solution is chosen as $S_{1}=2, T_{1}=1.043$, $S_{2}=0.895$, and $T_{2}=0.336$. The corresponding modal characteristic impedances are listed in Table II.

Fig. 6 shows the theoretical and measured results of filter $\alpha$. Quarter-wave transformers are used to match $Z_{o}=90 \Omega$ to $50 \Omega$ at the input and output ports. In Fig. 6, the curve denoted by "theoretical" is obtained by (11), and those by "present" and 
TABLE II

CHOSEN SOLUTIONS AND MODAL CHARACTERISTIC IMPEDANCES OF EACH COUPLED STAGE OF FILTERS $\alpha$ AND $\beta$

\begin{tabular}{l|l|l|l|c}
\hline \multicolumn{1}{c|}{ Filters } & \multicolumn{1}{c|}{ 1st stage } & \multicolumn{1}{c}{ 2nd stage } & 3rd stage & 4th stage \\
\hline$\alpha$ & $S_{1}=2$ & $S_{2}=0.895$ & & \\
$N=3$ & $T_{1}=1.043$ & $T_{2}=0.336$ & Same as the & Same as the 1st \\
$\Delta=30 \%$ & $Z_{o e 1}=136.93 \Omega$ & $Z_{o e 2}=55.40 \Omega$ & 2nd stage & stage \\
$Z_{o}=90 \Omega$ & $Z_{o o 1}=43.07 \Omega$ & $Z_{o o 2}=25.16 \Omega$ & & \\
\hline$\beta$ & $S_{1}=2$ & $S_{2}=0.96$ & $S_{3}=1.25$ & $S_{4}=1.65$ \\
$N=6$ & $T_{1}=1.23$ & $T_{2}=0.51$ & $T_{3}=0.4$ & $T_{4}=0.465$ \\
$\Delta=30 \%$ & $Z_{o o 1}=145.35 \Omega$ & $Z_{o o 2}=66.15 \Omega$ & $Z_{o o 3}=74.25 \Omega$ & $Z_{o o 4}=95.18 \Omega$ \\
$Z_{o}=90 \Omega$ & $Z_{o o 1}=34.65 \Omega$ & $Z_{o o 2}=20.25 \Omega$ & $Z_{o o 3}=38.25 \Omega$ & $Z_{o o 4}=53.33 \Omega$ \\
\hline
\end{tabular}

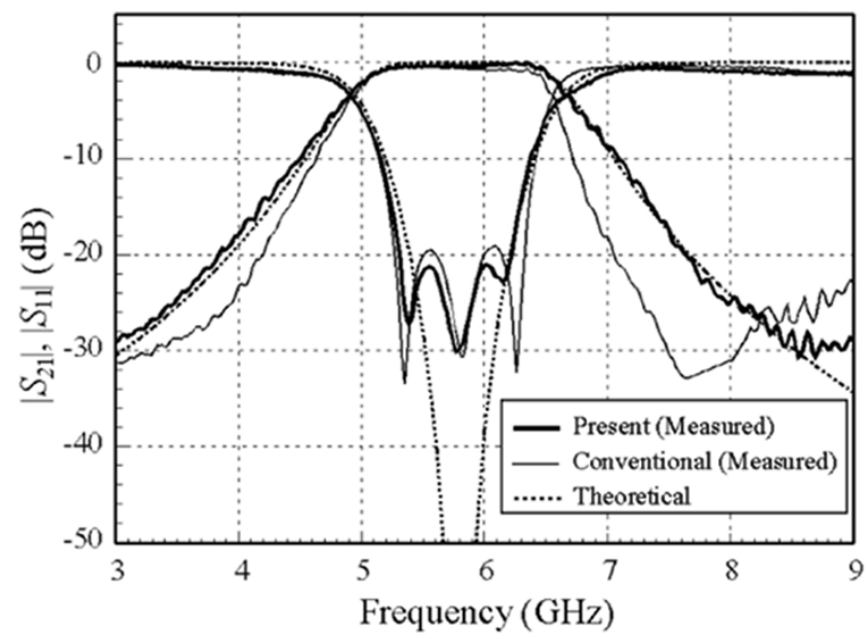

(a)

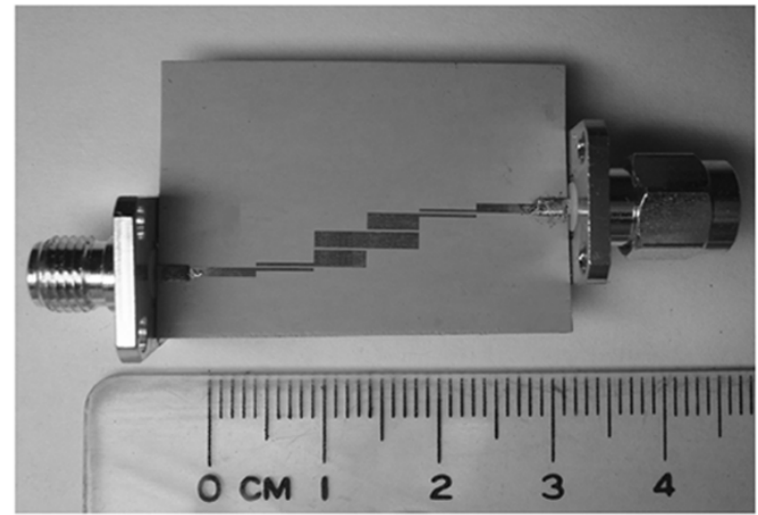

(b)

Fig. 6. (a) Theoretical and measured responses of filter $\alpha$. (b) Photograph of the fabricated circuit. $f_{o}=5.8 \mathrm{GHz}, N=3, \Delta=30 \%$. Circuit dimensions: $W_{1}=0.24 \mathrm{~mm}, G_{1}=0.15 \mathrm{~mm}, W_{2}=1.45 \mathrm{~mm}, G_{2}=0.13 \mathrm{~mm}$. The linewidth of the quarter-wave transformer is $0.8 \mathrm{~mm}$. Substrate: $\varepsilon_{r}=10.2$, thickness $=1.27 \mathrm{~mm}$

"conventional" are measured responses of filters synthesized by the present method and the conventional method [5], respectively. The "present" response matches with the "theoretical" maximally flat response very well. The excess poles of $\left|S_{11}\right|$ could result from the unequal even- and odd-mode phase velocities of the microstrip coupled stages. Detailed data show that

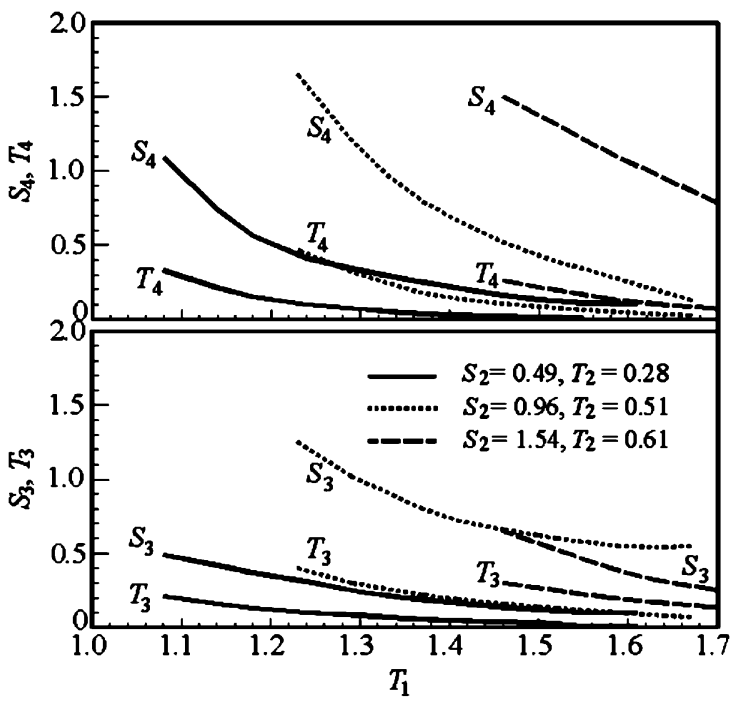

Fig. 7. Root loci of $S_{3}, T_{3}, S_{4}$, and $T_{4}$ for a sixth-order filter with $\Delta=30 \%$ for $\left(S_{2}, T_{2}\right)=(0.49,0.28),(0.96,0.51)$, and $(1.54,0.61)$.

the measured "present" filter has $\Delta=30.2 \%$, which is very close to the design. The filter based on the conventional method [4], [5] has $\Delta=26 \%$, mainly due to the use of frequency-independent J-inverters for the coupled line stages. This is consistent with the results reported in [6]. Fig. 6(b) presents a photograph of filter $\alpha$.

2) Filter $\beta$ : For a sixth-order filter, the $Q_{T}$ condition is

$$
\begin{aligned}
\left(2+S_{2}\right)\left(S_{2}+S_{3}\right)^{2}\left(S_{3}+S_{4}\right)^{2}[ & \left.\left(2+S_{2}\right)-T_{1}^{2}\right] \\
& =\left|\frac{\sin \theta}{\cos ^{6} \theta}\right| T_{1}^{2} T_{2}^{2} T_{3}^{2} T_{4} .
\end{aligned}
$$

There are three degrees of freedom for choosing the solutions. We take $T_{1}, S_{2}$, and $T_{2}$ as sweep variables in solving the simultaneous equations. If solutions with tough structural parameters are removed, the rest of $S_{2}$ ranges from 0.49 to 1.54 and $T_{2}$ from 0.28 to 0.61 for $1.1 \leq T_{1} \leq 1.7$. Three sets of solutions with $\left(S_{2}, T_{2}\right)=(0.49,0.28),(0.96,0.51)$, and $(1.54,0.61)$ are plotted in Fig. 7. Based on the design graph in Fig. 5 and $Z_{o}=$ $90 \Omega$, we choose a solution for filter $\beta$ for validating the circuit synthesis. As shown in Fig. 8, the simulation results match very well with the theoretical prediction. The simulated response has a BW of $30.3 \%$, i.e., only $0.3 \%$ away from the specification. The characteristic impedances for each coupled stage are listed in Table II and detailed dimensions are in the figure caption. Since some gap sizes, e.g., $G_{1}=0.06 \mathrm{~mm}$ and $G_{2}=0.02 \mathrm{~mm}$, are far beyond the best resolution of our fabrication facilities, only simulation responses are provided.

\section{IMPLEMENTATION USING TAPPED INPUT/OUTPUT}

In many cases, such as in Fig. 8, linewidths or gaps are too small to fabricate, even the degrees of freedom in choosing the solution are fully utilized. This situation becomes more severe when order or BW is increased. Fortunately, the tapped input/output [10], [11] can be used to resolve this problem. Theoretically, the tapping structure can realize a very wide range of the coupling coefficients. Thus, the criterion for choosing the 


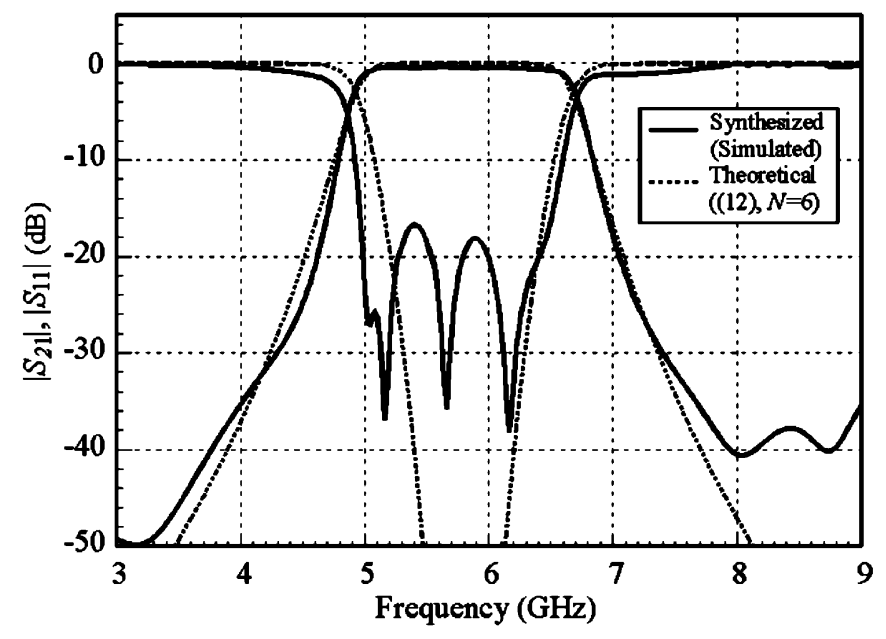

Fig. 8. Simulation and theoretical responses of filter $\beta . f_{o}=5.8 \mathrm{GHz}, N=$ $6, \Delta=30 \%$. Circuit dimensions: $W_{1}=0.24 \mathrm{~mm}, G_{1}=0.06 \mathrm{~mm}, W_{2}=$ $1.27 \mathrm{~mm}, G_{2}=0.02 \mathrm{~mm}, W_{3}=0.92 \mathrm{~mm}, G_{3}=0.47 \mathrm{~mm}, W_{4}=0.55 \mathrm{~mm}$, $G_{4}=0.78 \mathrm{~mm}$.

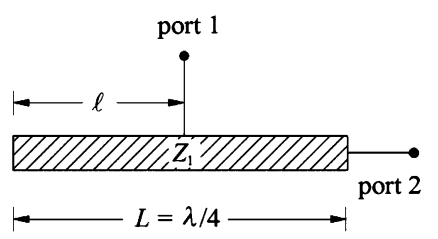

Fig. 9. Tapped line treated as a two-port network.

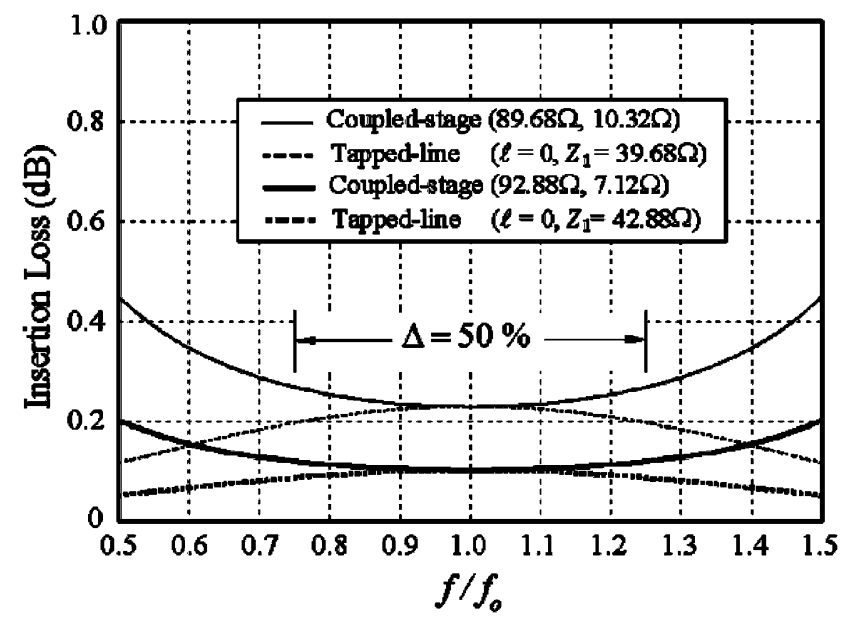

Fig. 10. Comparison of $\left|S_{21}\right|$ responses of tapped lines and coupled stages.

solution becomes to release dimensions of middle stages and locate the difficulties to the end stages as much as possible.

Since the derivation of the IL function (12) is based on a cascade of coupled stages, we have to establish the equivalence between a tapped resonator and a coupled stage. For the tapped structure in Fig. 9, let $\ell$ be the distance between the tap point and one end of the resonator and $Z_{1}$ be its characteristic impedance. It can be shown that its impedance matrix elements can be written as

$$
\begin{aligned}
& Z_{11}=-j Z_{1} \cos \beta \ell(\sin \beta \ell+\cos \beta \ell \cot \beta L) \\
& Z_{12}=Z_{21}=-j \frac{Z_{1} \cos \beta \ell}{\sin \beta L}
\end{aligned}
$$

TABLE III

CHOSEN ROOTS AND MODAL IMPEDANCES OF THE TWO EXPERIMENTAL FILTERS WITH TAPPED INPUTS

\begin{tabular}{l|l|l|c}
\hline \multicolumn{1}{c|}{ Filters } & \multicolumn{1}{c|}{$\mathbf{1}^{\text {st }}$ stage } & \multicolumn{1}{c}{ 2 $^{\text {nd }}$ stage } & 3 $^{\text {rd }}$ stage \\
\hline \hline $\boldsymbol{\delta}$ & $S_{1}=2$ & $S_{2}=2.629$ & \\
$N=3$ & $T_{1}=1.587$ & $T_{2}=1.112$ & \\
$\Delta=50 \%$ & $\ell=0$ & $Z_{o e 2}=93.53 \Omega$ & \\
$Z_{o}=50 \Omega$ & $Z_{1}=39.68 \Omega$ & $Z_{o o 2}=37.93 \Omega$ & \\
\hline$\gamma$ & $S_{1}=2$ & $S_{2}=2.619$ & $S_{3}=1.893$ \\
$N=5$ & $T_{1}=1.715$ & $T_{2}=1.11$ & $T_{3}=0.699$ \\
$\Delta=40 \%$ & $\ell=0$ & $Z_{o e 2}=93.23 \Omega$ & $Z_{o e 3}=64.80 \Omega$ \\
$Z_{o}=50 \Omega$ & $Z_{1}=42.88 \Omega$ & $Z_{o o 2}=37.73 \Omega$ & $Z_{o o 3}=29.85 \Omega$ \\
\hline
\end{tabular}

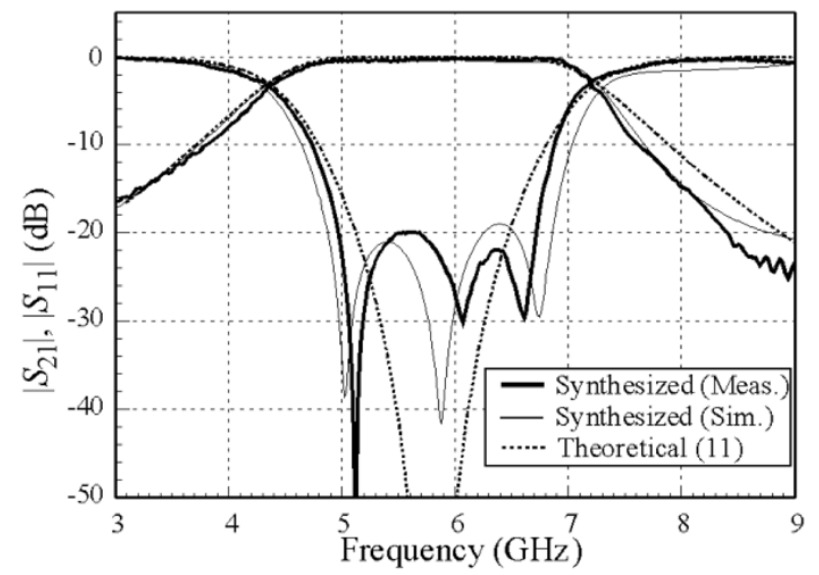

(a)

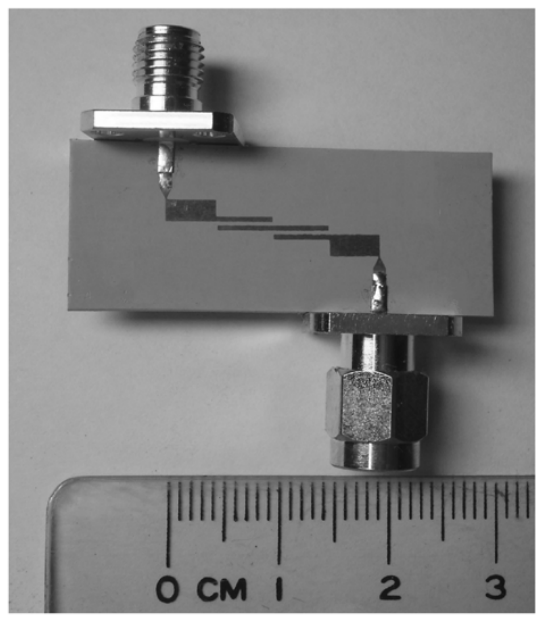

(b)

Fig. 11. (a) Theoretical, simulated, and measured responses of filter $\delta . f_{o}=$ $5.8 \mathrm{GHz}, N=3$, and $\Delta=50 \%$. (b) Photograph of the fabricated circuit. Circuit dimensions: $W_{1}=2 \mathrm{~mm}, W_{2}=0.54 \mathrm{~mm}, G_{2}=0.23 \mathrm{~mm}$.

$$
Z_{22}=-j Z_{1} \cot \beta L
$$

At the same time, the $Z$ matrix elements of a coupled line stage are in (2). The equivalence of these two two-ports can be established by letting $\theta=\beta L=\pi / 2, \ell=0$, and $Z_{1}=\left(Z_{o e}-\right.$ $\left.Z_{o o}\right) / 2$. The equivalence is, however, valid only for a finite frequency band. Fig. 10 investigates the performance of the equivalence. Two coupled stages with $\left(Z_{o e}, Z_{o o}\right)=(92.88 \Omega, 7.12 \Omega)$ and $(89.68 \Omega, 10.32 \Omega)$ are studied. The sum of $Z_{o e}$ and $Z_{o o}$ is $100 \Omega$ since $S_{1}=2$ and $Z_{o}=50 \Omega$ is expected. In Fig. 10, both 


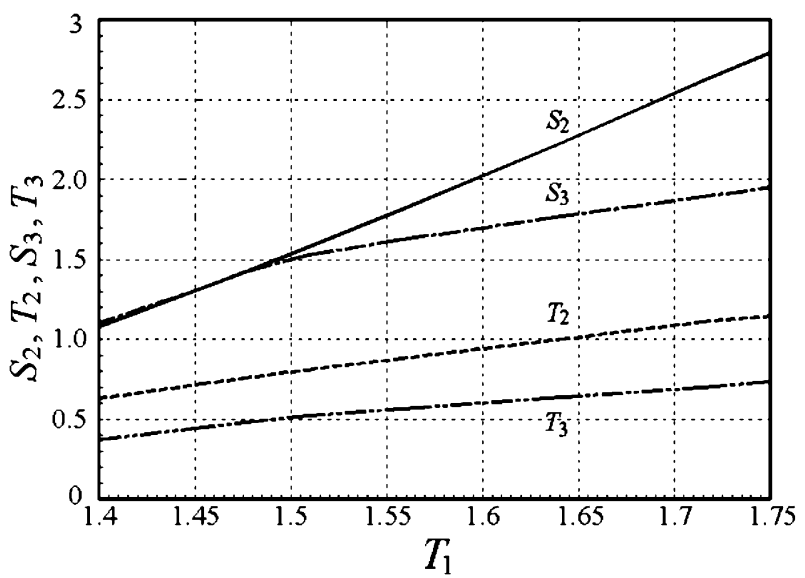

Fig. 12. Possible roots for the second and third coupled stages of a fifth-order parallel-coupled line filter with $\Delta=40 \%$.

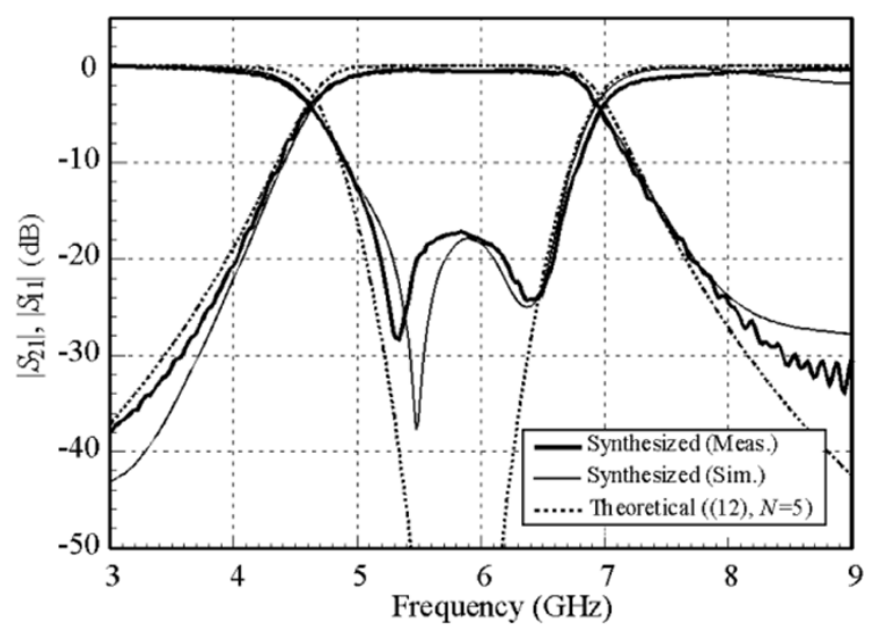

(a)

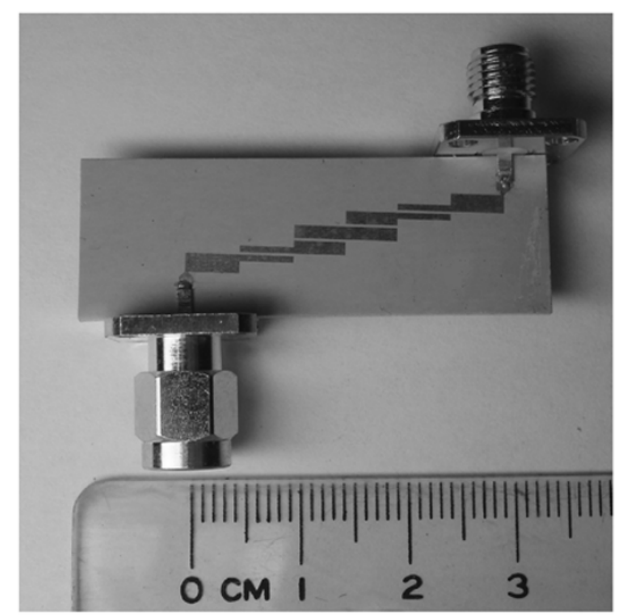

(b)

Fig. 13. (a) Theoretical, simulated, and measured responses of filter $\gamma . f_{o}=$ $5.8 \mathrm{GHz}, N=5$, and $\Delta=40 \%$. (b) Photograph of the fabricated circuit. Circuit dimensions: $W_{1}=1.7 \mathrm{~mm}, W_{2}=0.58 \mathrm{~mm}, W_{3}=1.2 \mathrm{~mm}, G_{2}=$ $0.22 \mathrm{~mm}, G_{3}=0.22 \mathrm{~mm}$.

cases have a maximal $\left|S_{21}\right|$ deviation less than 0.08 and $0.34 \mathrm{~dB}$ within a BW of $50 \%$ and $100 \%$, respectively. These two tapped line structures will be employed to the following two experimental filters.

1) Filter $\delta$ : This filter is designed to have $N=3$ and $\Delta=$ $50 \%$. Based on (15), $\theta=1.1781 \mathrm{rad}$, the $S_{2}$ and $T_{2}$ solutions for $T_{1}$ varying from 1.1 to 1.6 are shown in Fig. 4. For realization, the chosen roots and modal characteristic impedances are listed in Table III with $Z_{o}=50 \Omega$. A tapped resonator with $Z_{1}=39.68 \Omega$ is used to replace the end stages with $Z_{o e 1}=89.68 \Omega$ and $Z_{o o 1}=10.32 \Omega$. Fig. 11(a) plots the theoretical, simulated, and measured responses. They have very good agreement within the passband. Detailed data show that the BWs of the simulated and measured results have only $0.5 \%$ and $-0.5 \%$, respectively, away from the theory. The measured midband IL is approximately $0.35 \mathrm{~dB}$. A photograph of the fabricated filter is shown in Fig. 11(b). Note that the line gap $0.23 \mathrm{~mm}$ is much easier to realize than the $0.13-\mathrm{mm}$ gap of filter $\alpha$. Thus, as compared with filter $\alpha$, there are at least two advantages incorporating the tapped input/output into the design. One is that it greatly releases the tough circuit dimensions even though the $\mathrm{BW}$ is increased from $30 \%$ to $50 \%$, and the other is that the impedance transformer can be saved since $Z_{o}=50 \Omega$.

2) Filter $\gamma$ : The second experiment is a fifth-order filter with $\Delta=40 \%$. Fig. 12 plots the filtered roots with $\theta=1.2566 \mathrm{rad}$. The end stages, with $Z_{o e 1}=92.88 \Omega$ and $Z_{o o 1}=7.12 \Omega$, are replaced with a tapped resonator with $Z_{1}=42.88 \Omega$. Fig. 13(a) plots the theoretical, simulation, and measured results. All of them show good agreement. The measured midband IL is $0.5 \mathrm{~dB}$. The BW of the measured response has approximately $1 \%$ less than the theoretical calculation by (12b). The required minimal gap of this filter is $0.22 \mathrm{~mm}$. If a coupled stage is used instead, the required line gap will be less than $0.01 \mathrm{~mm}$. Fig. 13(b) shows the photograph of the experiment circuit.

\section{CONCLUSIONS}

Parallel coupled line filters with maximally flat responses of order $N \leq 6$ are synthesized based on derived IL functions. Simultaneous equations for maximally flat responses and the $Q_{T}$ condition are formulated for determining $Z_{o e}$ and $Z_{o o}$ of each coupled stage. The under-determined conditions leave several degrees of freedom in choosing the circuit dimensions. By properly utilizing these degrees of freedom, the problem resulted from the tight coupled line dimensions can be resolved by gathering all difficulties to the end stages and employing tapped input/output to replace the end stages. Four circuits are simulated and three of them are fabricated and measured to demonstrate the formulation and circuit synthesis. The measured results manifest very accurate BWs and show that the proposed method not only provide a significant improvement in predicting the filter BW, but also preserve the quality of passband responses.

\section{REFERENCES}

[1] S. B. Cohn, "Parallel-coupled transmission-line resonator filters," IRE Trans. Microw. Theory Tech., vol. MTT-6, no. 4, pp. 223-231, Apr. 1958

[2] L. Zhu, W. Menzel, K. Wu, and F. Boegelsack, "Theoretical characterization and experimental verification of a novel compact broad-band microstrip bandpass filter," in Proc. Asia-Pacific Microwave Conf., 2001, pp. 625-628. 
[3] C.-Y. Chang and T. Itoh, "A modified parallel-coupled filter structure that improves the upper stopband rejection and response symmetry," IEEE Trans. Microw. Theory Tech., vol. 39, no. 2, pp. 310-314, Feb. 1991.

[4] G. L. Matthaei, L. Young, and E. M. T. Johns, Microwave Filters, Impedance Matching Networks, and Coupling Structures. Norwood, MA: Artech House, 1980.

[5] D. M. Pozar, Microwave Engineering, 2nd ed. New York: Wiley, 1998.

[6] K.-S. Chin, L.-Y. Lin, and J.-T. Kuo, "New formulas for synthesizing microstrip bandpass filters with relatively wide bandwidths," IEEE Microw. Wireless Compon. Lett., vol. 14, no. 5, pp. 231-233, May 2004.

[7] W. W. Mumford, "Tables of stub admittances for maximally flat filters using shorted quarter-wave stubs," IEEE Trans. Microw. Theory Tech., vol. MTT-13, no. 9, pp. 695-696, Sep. 1965.

[8] J. M. Drozd and W. T. Joines, "Maximally flat quarter-wavelength-coupled transmission-line filters using $Q$ distribution," IEEE Trans. Microw. Theory Tech., vol. 45, no. 12, pp. 2100-2113, Dec. 1997.

[9] R. Levy, "Theory of direct coupled cavity filters," IEEE Trans. Microw. Theory Tech., vol. MTT-15, no. 6, pp. 340-348, Jun. 1967.

[10] E. G. Cristal, "Tapped-line coupled transmission lines with applications to interdigital and combline filters," IEEE Trans. Microw. Theory Tech., vol. MTT-23, no. 12, pp. 1007-1012, Dec. 1975.

[11] J. S. Wong, "Microstrip tapped-line filter design," IEEE Trans. Microw. Theory Tech., vol. MTT-27, no. 1, pp. 44-50, Jan. 1979.

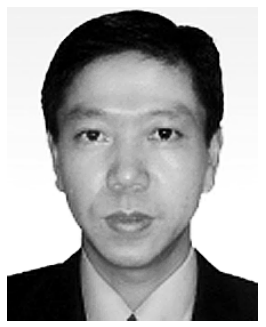

Kuo-Sheng Chin received the M.S.E.E. degree from Syracuse University, Syracuse, NY, in 1993, and is currently working toward the Ph.D. degree in communication engineering at the National Chiao Tung University (NCTU), Taiwan, R.O.C.

Since 1986, he has been with the Chung Shan Institute of Science and Technology (CSIST), Taoyuan, R.O.C., and has been involved with radomes and antennas research. He is currently a Senior Scientist with CSIST. His main research interests are microwave measurements and microwave circuit design.

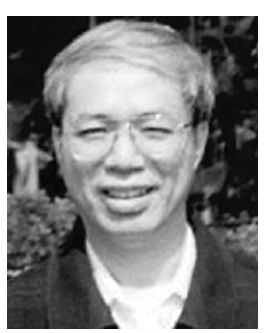

Jen-Tsai Kuo (S'88-M'92-SM'04) received the $\mathrm{Ph} . \mathrm{D}$. degree from the Institute of Electronics, National Chiao Tung University (NCTU), Taiwan, R.O.C., in 1992.

Since 1984, he has been with the Department of Communication Engineering, NCTU, as a Lecturer in both the Microwave and Communication Electronics Laboratories. During 1995 academic year, he was a Visiting Scholar with the University of California at Los Angeles (UCLA). He is currently a Professor with the Department of Communication Engineering, NCTU, and serves as the Chairman of the Degree Program of the Electrical Engineering and Computer Science (EECS) College, NCTU. His research interests include the analysis and design of high-frequency electronics and microwave circuits, high-speed interconnects and packages, field-theoretical studies of guided waves, and numerical techniques in electromagnetics. 